\section{Estudo clínico, randomizado, duplo- cego, em crianças com adenóide obstrutiva, submetidas a tratamento homeopático}

\author{
Sergio E. Furuta ${ }^{1}$, Luc L.M. Weckx², \\ Claudia R. Figueiredo ${ }^{3}$
}

bjetivo: Avaliar a eficácia e segurança do tratamento homeopático em crianças com adenóide obstrutiva, com indicação cirúrgica. Forma de estudo: Clínico prospectivo. Material e método: Estudo prospectivo, duplo-cego, randomizado, em que foram incluídas 40 crianças com idade variando de 3 a 7 anos, 20 crianças foram tratadas com medicação homeopática individualizada (Simillimum), baseada no princípio da similitude e 20 crianças receberam placebo. Todas as crianças do grupo medicação homeopática foram medicadas diariamente com Agraphis nutans $6 \mathrm{CH}$, Thuya $6 \mathrm{CH}$ e Adenóide $21 \mathrm{CH}$; e as do grupo placebo receberam diariamente medicamentos sem 0 princípio ativo. A duração do estudo de cada paciente foi de 4 meses. A avaliação dos resultados foi clínica, por meio de questionário padrão, de exame otorrinolaringológico e nasofaringoscopia direta com fibroscópio flexível, no primeiro e no último dia de tratamento. Utilizou-se como critério de inclusão a adenóide que ocupou mais do que $70 \%$ da luz coanal. Resultados: Das 20 crianças tratadas com medicamento homeopático, 13 não apresentaram alteração no tamanho da adenóide nos exames nasofaringoscópicos e 7 tiveram diminuição da adenóide; das 20 crianças que receberam placebo por 4 meses, 11 não apresentaram alterações no tamanho da adenóide, 4 tiveram diminuição da adenóide e 5 crianças tiveram aumento. Não houve diferença estatística significante entre os grupos $(P=0,069)$. Na avaliação clínica da evolução dos pacientes, dos 20 pacientes tratados com medicamento homeopático, 17 se mantiveram inalterados, com respiração oral e ronco, um paciente melhorou, ficando sem ronco e dois foram curados, isto é, a respiração alterou-se de oral para nasal e sem ronco. Dos 20 pacientes tratados com placebo, 17 pacientes se mantiveram inalterados, um paciente melhorou do ronco e dois foram curados, não tendo havido diferença estatística significante entre os grupos ( $P>0,999)$. Conclusões: 0 tratamento homeopático não foi eficaz nas crianças com adenóide obstrutiva, mantendo-se a indicação cirúrgica em $85 \%$ dos pacientes. 0 medicamento homeopático não provocou eventos adversos nas crianças.

\section{Prospective, randomized, double-blind clinical trial about efficacy of homeopathic treatment in children with obstructive adenoid}

\author{
Palavras-chave: adenóide obstrutiva, tratamento \\ homeopático. \\ Key words: adenoid, homeopathic treatment.
}

\begin{abstract}
summary
1 im: the efficacy and security of homeopathic treatment was investigated on children with obstructive adenoid justifying an operation. Study design: Clinical prospective. Material and method: In a prospective, randomized, double-blind clinical trial included 40 children between the ages of 3 to 7 years old, 20 children were treated with homeopathic medication, based in the principle of similarity (Simillimum), and 20 children with placebo. All the children of the homeopathic group/ adenoid, were treated daily with Agraphis nutans $6 \mathrm{CH}$, Thuya $6 \mathrm{CH}$ and Adenoid $21 \mathrm{CH}$, and the patients of the placebo group received daily placebo medication. The duration of the study of each children was 4 months. The evaluation of the results was clinical, and it was made by questionnaire standard, clinical examination and direct flexible fiberoptic nasopharyngoscopy, in the first and last day of treatment. The criterion of selection was the adenoid that occuped more than $70 \%$ of the coanal space. Results: From the group of 20 children treated with homeopathic treatment, 13 did not show any change on the size of adenoid after nasopharyngoscopy, and 7 children had their adenoid decreased; from another group of 20 children that have treated with placebo for 4 months, 11 did not show any change on the size of their adenoid, 4 had their adenoid decreased and 5 had their adenoid increased. The statistical analysis showed a not significant difference $(P=0,069)$. The clinical evaluation of the patients showed that from the group of 20 patients treated with homeopathy, 17 kept unchanging, with oral breathing and snoring, one patient got better, eliminating the snoring and two were cured, which mean that their oral breathing turned to nasal breathing without snoring. From the group of 20 patients treated with placebo, 17 kept unchanging, one eliminated the snoring and two were cured; and these differences were not statistically significant $(P>0,999)$. Conclusions: the homeopathic treatment was not efficient in the patients with obstructive adenoid, remaining it surgical indication in $85 \%$ of the children. The homeopathic remedies did not provoke adverse events in the children.
\end{abstract}

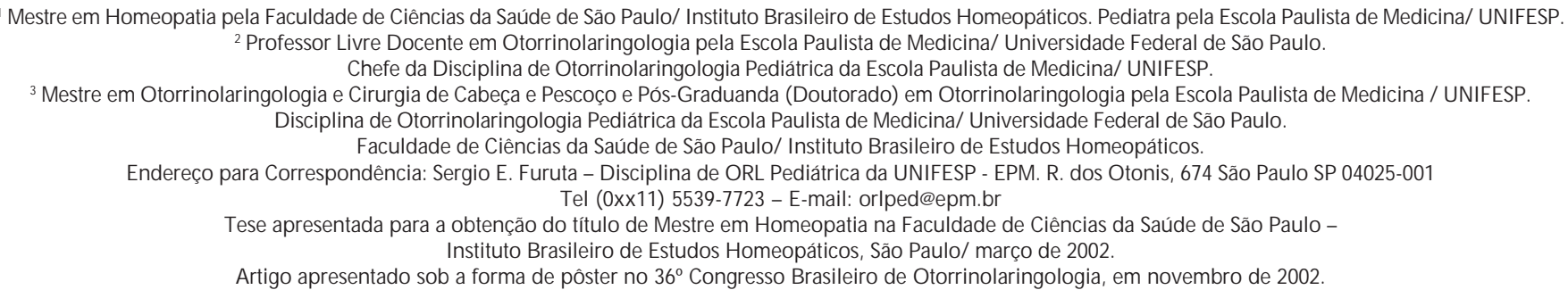




\section{INTRODUÇÃO}

Uma causa freqüente de consulta ao pediatra e ao otorrinolaringologista e uma das indicações cirúrgicas mais comuns na especialidade são as hipertrofias das tonsilas palatinas e faríngea. Na primeira metade do século, a amigdalectomia e a adenoidectomia chegaram a ser indicadas aos mínimos sintomas, até como cirurgia de rotina para quase todas as doenças de crianças, inclusive retardo mental e enurese ${ }^{1}$. A partir dos anos 60, estudos mostraram a ineficácia da cirurgia em muitos casos, surgindo dúvidas sobre a indicação cirúrgica. Nessa época, iniciaram-se pesquisas sobre o papel imunológico das estruturas do anel linfático de Waldeyer, tornando a indicação cirúrgica mais conservadora e criteriosa ${ }^{1}$.

A Homeopatia, criada pelo médico alemão Samuel Hahnemann em 1796, tem sido utilizada com sucesso na prevenção e tratamento das doenças das tonsilas palatinas e faríngea, reduzindo o número de pacientes com indicação cirúrgica². Além disso, o custo do medicamento homeopático é muito inferior ao do alopático. Entretanto, a literatura a respeito da eficácia do medicamento homeopático é escassa, principalmente com metodologia científica adequada, utilizando o método duplo-cego, randomizado e o placebo.

Homeopatia (grego homolós $=$ semelhante + páthos $=$ doença ou sofrimento) designa a ciência terapêutica baseada na lei natural de cura Similia similibus curentur (semelhantes curados pelos semelhantes) ${ }^{3}$. Representa um método terapêutico que permite um confronto de semelhança entre os sintomas de um doente com os obtidos pela experimentação de uma substância em indivíduos aparentemente sadios e sensíveis. Essa substância, medicamento homeopático, recebeu a denominação de Simillimum ou remédio "de fundo" e farmacologicamente é preparada sob a forma de ultradiluição ${ }^{4}$.

Os princípios da Homeopatia são: 1. Lei da semelhança; 2. Experimentação no homem sadio (as experimentações das drogas não devem ser em doentes ou animais, mas em homens sadios e sensíveis; o conjunto de manifestações apresentadas durante a experimentação recebe o nome de patogenesia); 3. Remédio único (utilizar um único medicamento, o Simillimum); 4. Dose mínima (medicamento diluído e dinamizado) ${ }^{5}$.

Os medicamentos homeopáticos não são simples diluições de uma substância ao infinito. Cada vez que 0 medicamento é diluído, é agitado vigorosamente, mecanismo denominado sucussão. Acredita-se que esta agitação "desperta" uma energia medicamentosa latente (dinamização). Provém dos três reinos: vegetal, mineral e animal. A preparação medicamentosa geralmente é feita nas escalas decimal e centesimal. Na escala decimal dilui-se uma parte da substancia básica em 9 partes de um veículo e agita-se (sucussão) 100 vezes (1 D). o veículo geralmente é o álcool a 30\%. Na centesimal a diluição é feita com uma parte da substância básica em 99 partes de veículo e sucussiona-se 100 vezes (1 $\mathrm{CH}=1$ Centesimal Hahnemaniana).

Define-se medicamento isopático, segundo a Farmacopéia Homeopática Brasileira ${ }^{6}$, como preparações medicamentosas de uso homeopático obtidas a partir de produtos biológicos, quimicamente indefinidos, secreções, excreções, tecidos e órgãos, patológicos ou não, produtos de origem microbiana e alérgenos. Segundo Costa7 ${ }^{7}$ Isopatia é o méto do de curar as doenças por meio dos seus agentes causais manipulados mediante técnica homeopática (dinamizados). 0 autor preconiza a associação da homeopatia e isopatia nos tratamentos, utilizando três medicamentos para um mesmo paciente:

a) Medicamento baseado na totalidade sintomática do paciente, o remédio constitucional, ou "de fundo", ou "sósia" medicamentoso homeopático.

b) Remédio Episódico ou Sindrômico, isto é, aquele que corresponde aos sintomas agudos, mais incomodativos, geralmente localizados. Normalmente um remédio específico para a patologia física, dentro de uma conduta organicista.

c) Medicamento Etiopatogenico e/ou Fisiopatologico da doença; conforme o caso clínico a saber:

c1) Nosódios - isopáticos não lisados ou destruídos, e/ou vivos específicos, dinamizados, autógenos.

c2) Alérgenos ou mediadores dinamizados

c3) Organoterápicos dinamizados

Denomina-se Nosódio (grego noso: doença) o medicamento preparado segundo a farmacotécnica homeopática, com produtos patológicos vegetais ou animais. $\mathrm{Na}$ França são denominados Bioterápicos ${ }^{7}$.

Este estudo, duplo-cego, randomizado, visou avaliar a eficácia e segurança do tratamento homeopático em crianças com indicação cirúrgica de adenoidectomia por hipertrofia obstrutiva de tonsila faríngea.

\section{MATERIAL E MÉTODO}

Foram selecionados 40 pacientes do ambulatório da Disciplina de O torrinolaringologia Pediátrica da Escola Paulista de Medicina/ Universidade Federal de São Paulo (UNIFESP) e do Hospital São Paulo, no período de março/2000 a setembro/2001, com faixa etária de 3 a 7 anos, com indicação cirúrgica por adenóide obstrutiva, aguardando vaga para cirurgia. Excluíram-se deste estudo pacientes com doenças sistêmicas e os com imunodeficiência. A adenóide obstrutiva, nos respiradores bucais, com ou sem ronco noturno, e/ ou apnéia, foi caracterizada e avaliada ao exame, por nasofibroscopia realizada pelo otorrinolaringologista. $\mathrm{Na}$ nasofibroscopia foi considerada obstrutiva a adenóide que ocupou mais do que $70 \%$ da luz coanal ${ }^{8}$. 
A amostra foi dividida e randomizada em 2 grupos duplo-cegos:

Grupo I - 20 crianças com diagnóstico de adenóide obstrutiva, submetidas a tratamento homeopático por 4 meses.

Grupo II - 20 crianças com diagnóstico de adenóide obstrutiva, submetidas a tratamento com placebo, por 4 meses.

Os pais ou responsáveis pelo paciente foram informados do objetivo do estudo e obtido o consentimento escrito dos mesmos. Este estudo foi aprovado pelo Comitê de Ética em Pesquisa da Universidade Federal de São Paulo (UNIFESP) - EPM.

O tratamento homeopático constou da administração de 3 medicamentos, para todos os pacientes do grupo I, baseado na experiência pessoal, de Costa ${ }^{7}$ e Linhares²:

1) Medicamento constitucional do paciente, também conhecido homeopaticamente como "Simillimum" ou "de fundo", individualizado, baseado nas semelhanças físicas e mentais do paciente, obtidas na anamnese, na potência de $30 \mathrm{CH}$ em dose única, reavaliado a cada 4 semanas, por 4 meses. Utilizou-se o programa de informática Repertório Homeopático Digital $1{ }^{9}$ para auxiliar na determinação do medicamento Simillimum.

2) Medicamento baseado nas características orgânicas, locais, da adenóide, Agraphis nutans, na potência $6 \mathrm{CH}$ e Thuya $6 \mathrm{CH}$ diariamente, por 4 meses.

3) Medicamento isopático, Adenóide $21 \mathrm{CH}$, diariamente, por 4 meses.

Os pacientes do grupo II receberam placebo como se fosse o medicamento constitucional do paciente em dose única, placebo como se fosse o medicamento Agraphis nutans diariamente, placebo como se fosse o medicamento Thuya diariamente e placebo como se fosse o medicamento Adenóide diariamente, por 4 meses. A listagem dos medicamentos randomizados foi feita pela farmacêutica homeopata, responsável pela confecção dos medicamentos e só foi divulgada após o término do tratamento de todos os pacientes. 0 placebo foi constituído por álcool a 30\%, com mesmo odor, sabor e aspecto do medicamento homeopático ativo. A função do álcool é o de conservante. Todos os medicamentos utilizados neste estudo estão de acordo com a Farmacopéia Homeopática Brasileira (1997) ${ }^{6}$.

A avaliação clínica constou de questionário padrão, com acompanhamento mensal por quatro meses. Todos os pacientes foram submetidos a avaliação otorrinolaringológica que constou de exame otorrinolaringológico (oroscopia, rinoscopia anterior e otoscopia) e nasofibroscopia, no primeiro e no último dia de tratamento, por um otorrinolaringologista da Disciplina de Otorrinolaringologia Pediátrica da Escola Paulista de Medicina/UNIFESP. No final do estudo, todos os pacientes que mantiveram a indicação cirúrgica foram encaminhados para a cirurgia.

No método estatístico foi aplicado o teste exato de Fisher ou uma extensão para Tabelas maiores que $2 \times 2$, tendo-se fixado como nível de significância o valor 0,05 ou $5 \%$, assinalando-se com um asterisco $(*)$ os valores significantes.

\section{RESULTADOS}

Foram recrutadas 17 crianças do sexo feminino (43\%) e 23 do sexo masculino (57\%), com idades variando de 3 a 7 anos. Não houve nenhuma desistência durante 0 tratamento. Pelo princípio da similitude, foram receitados, individualmente, um dos medicamentos homeopáticos: Lycopodium, Nux-vomica, Ignatia, Lachesis, Natrum muriaticum, Pulsatilla, Carcinosinum, Phosphorus e Sépia (Gráfico 1).

Os resultados dos exames nasofibroscópicos mostraram que não houve alteração no tamanho das adenóides (resultado inalterado) em 13 pacientes do grupo I (medicamento homeopático) e em 11 pacientes do grupo II (placebo). Constatou-se uma diminuição das adenóides (resultado melhor) em 7 pacientes do grupo I (medicamento homeopático) e em 4 pacientes do grupo II (placebo). Houve aumento das adenóides (resultado pior) em 5 pacientes do grupo II (placebo), conforme a Tabela 1 e

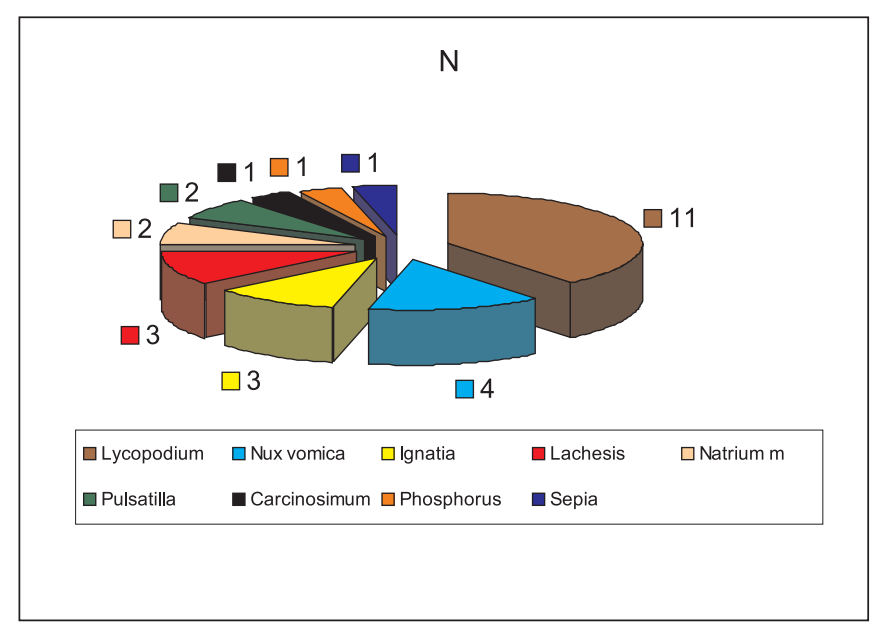

Gráfico 1. Medicamentos prescritos / Adenóide obstrutiva / Grupo I

Tabela 1. Adenóide obstrutiva / Evolução dos pacientes, comparando nasofibroscopia Inicial e final do tratamento

\begin{tabular}{ccccc}
\hline Grupo & Pior & Inalterado & Melhor & Total \\
\hline Placebo(II) & 5 & 11 & 4 & 20 \\
Medicação(I) & 0 & 13 & 7 & 20 \\
\hline Total & 5 & 24 & 11 & 40 \\
\hline
\end{tabular}

Teste exato de Fisher: $\mathrm{P}=0,069$ ou $6,9 \%$ 
Gráficos 2, 3, 4. Não houve significância na analise estatística dos grupos I e II ( $P=0,069$ ou $6,9 \%)$.

A avaliação clínica da evolução dos pacientes, considerando-se a respiração, no grupo I (medicamento homeopático) 17 pacientes se mantiveram inalterados, com respiração oral e ronco, 1 paciente melhorou, ficando sem ronco e 2 foram curados, isto é, a respiração alterou-se de oral para nasal e sem ronco, segundo a Tabela 2 e Gráfico 5. No grupo II (placebo) 17 pacientes se mantiveram inalterados, 1 paciente melhorou do ronco e 2 foram curados. Não houve diferença estatística significante entre os grupos I e II ( $P>0,999$ ou $9,99 \%)$.

Não foram constatados eventos adversos nos pacientes dos grupos I e II, relativos à utilização dos medicamentos.

\section{DISCUSSÃO}

A Homeopatia tem sido utilizada como uma alternativa na terapia das Adenóides obstrutivas, com a finalidade de diminuir as indicações cirúrgicas. A dificuldade na utilização do método duplo-cego randomizado nas pesquisas

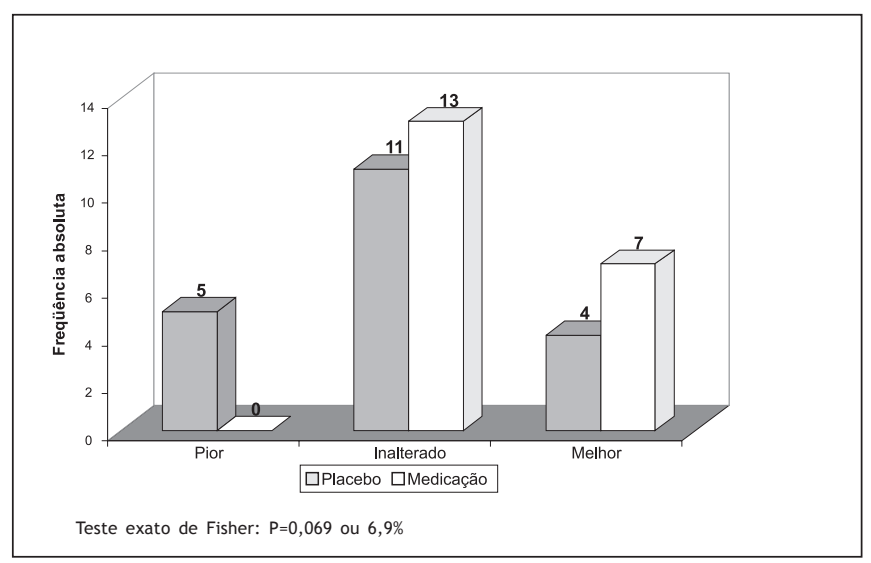

Gráfico 2. Distribuição dos pacientes segundo evolução e grupo de estudo Avaliação nasofibroscópica inicial e final / Adenóide obstrutiva homeopáticas está na característica da Homeopatia em individualizar cada paciente, determinando pelo princípio da similitude, o medicamento específico ("Simillimum"), independente do diagnóstico clínico em questão. A Homeopatia trata o doente como um todo, não apenas um sintoma ou uma doença. Assim, para o diagnóstico clínico de Adenóide obstrutiva foram selecionados 9 medicamentos homeopáticos distintos, individualizados. 0 atendimento de todos os pacientes foi realizado por um único médico.

Tabela 2. Evolução dos pacientes / Avaliação clínica / Adenóide obstrutiva

\begin{tabular}{ccc}
\hline Análise Clínica & Placebo & Medicamento \\
\hline Inalterado & 17 & 17 \\
Sem ronco & 1 & 1 \\
Curado & 2 & 2 \\
\hline Total & 20 & 20 \\
\hline
\end{tabular}

Teste exato de Fisher: $P>0,999$ ou $9,99 \%$

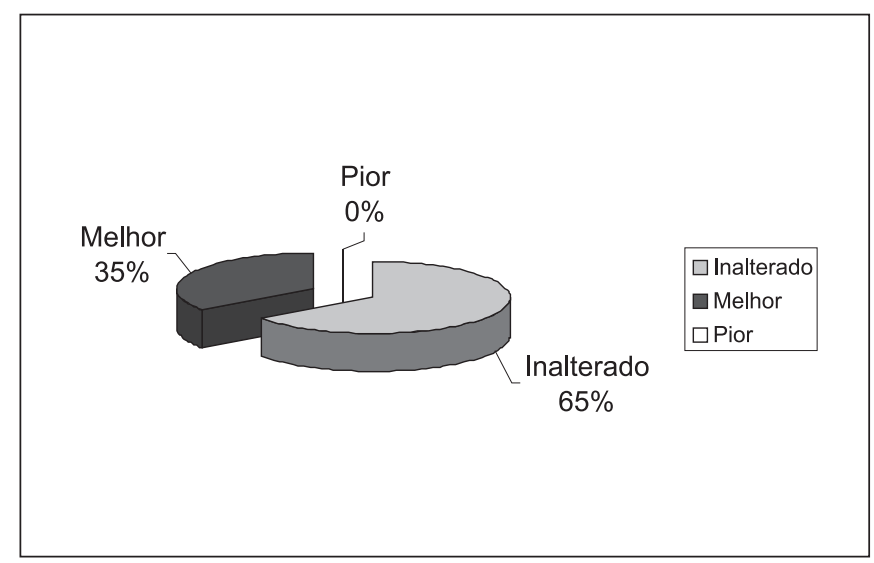

Gráfico 3. Evolução dos pacientes, grupo I (medicamento homeopático) comparando nasofibroscopia inicial e final do tratamento

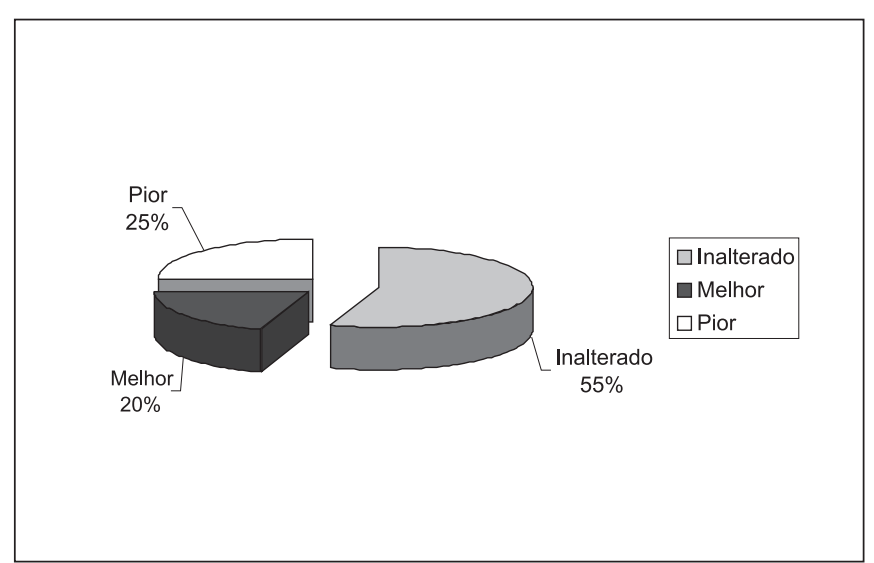

Gráfico 4. Evolução dos pacientes, grupo II (placebo), comparando nasofibroscopia inicial e final do tratamento

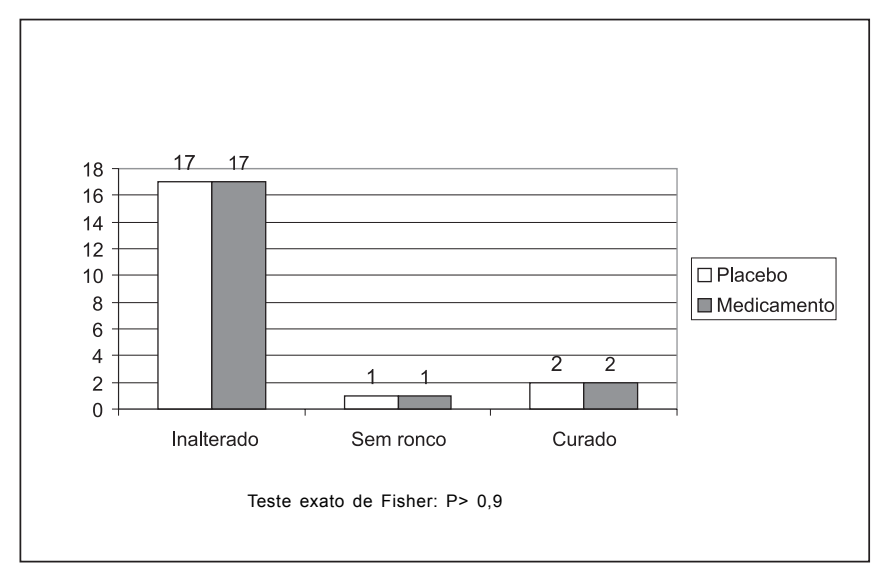

Gráfico 5. Distribuição dos pacientes segundo evolução e grupo de estudo / Avaliação clínica / Adenóide obstrutiva 
Comparando os resultados da nasofibroscopia no início e no final do tratamento, no grupo I (medicamento homeopático), 7 (35\%) melhoraram e 13 (65\%) ficaram inalterados. No grupo II (placebo), 4 (20\%) melhoraram, 11 (55\%) ficaram inalterados e 5 (25\%) pioraram. Não houve significância estatística. A avaliação clínica da evolução dos pacientes dos grupos I e II, analisando-se 0 tipo de respiração, se oral ou nasal, presença de ronco, não apresentou diferença significante. 17 pacientes (85\%) dos grupos I e II continuaram com indicação cirúrgica. Estes resultados estão de conformidade com os obtidos por Friese et al. ${ }^{10}$ que trataram 82 crianças, de 4 a 10 anos de idade, com adenóide obstrutiva, com indicação cirúrgica, com duração de 3 meses por paciente, num estudo duplo-cego, randomizado. No final do tratamento $70,7 \%$ das crianças tratadas com placebo e $78,1 \%$ das crianças tratadas com medicamento homeopático não necessitaram de cirurgia, concluindo-se que não houve significância estatística.

\section{CONCLUSÕES}

Dos resultados obtidos pela avaliação clínica e nasofibroscópica de 40 pacientes com adenóide obstrutiva com indicação cirúrgica, com 3 a 7 anos de idade, submetidos a tratamento homeopático ou placebo, duplo-cego, randomizado, com acompanhamento por 4 meses, pode-se concluir que:

1) 0 tratamento homeopático não foi eficaz nos pacientes com adenóide obstrutiva, mantendo-se em $85 \%$ dos pacientes, a indicação cirúrgica.

2) 0 medicamento homeopático não provocou efeitos colaterais nos pacientes.

\section{AGRADECIMENTOS}

Ao professor de Homeopatia, Dr. Carlos Roberto Dias Brunini pelo apoio e orientações.

À farmacêutica homeopata Andréa Cristina de O liveira, da Farmácia Núcleo da Manipulação pelo fornecimento dos medicamentos e listagem dos placebos.

Aos Pós-graduandos e Especializandos da Disciplina de ORL Pediátrica da UNIFESP/EPM pelas consultas e exames otorrinolaringológicos deste trabalho.

\section{REFERÊNCIAS BIBLIOGRÁFICAS}

1. Rosenfeld RM, Green RP. Tonsillectomy and adenoidectomy: changing trends. Ann Otol Rhinol Laryngol 1990;99:187-91.

2. Linhares W. Homeopatia em pediatria. $4^{a}$ ed. São Paulo: Homeolivros; 2000. 109 p.

3. Hahnemann S. O moderno Organon da arte de curar. Tradução Marcelo Pustiglione. 6a ed. Alemã. São Paulo: Typus; 2001. 320 p.

4. Kossak-Romanach A. Homeopatia em 1000 conceitos. São Paulo: Elcid; 1984. 27, 170.

5. Eizayaga FX. Tratado de medicina homeopática. Buenos Aires: Marecel; 1992. 400p.

6. Farmacopéia Homeopática Brasileira. 2a ed. parte I. São Paulo: Atheneu; 1997.

7. Costa RA. Homeopatia atualizada: Escola brasileira. $3^{a}$ ed. Petrópolis: Vozes; 1988. 78, 80, 94, 145-6.

8. Chami FI. Avaliação nasofibroscópica e radiológica de pacientes com hiperplasia de amígdala faríngea. São Paulo, 1997. [Mestrado] Universidade Federal de São Paulo - Escola Paulista de Medicina. $57 \mathrm{p}$.

9. Ribeiro Filho A, Bronfman Z. Repertório Homeopático Digital II São Paulo: Organon 2000. CD-ROM.

10. Friese KH, Feuchter U, Moeller H. Die homoopathische behandlung von adenoiden vegetationen. Ergebnisse einer prospektiven randomisierten Doppelblindstudie. HNO $1997 ; 45(8): 618-24$. 